\title{
Structural Properties of Pore-Forming Oligomers of $\alpha$-Synuclein
}

\author{
Hai-Young Kim, ${ }^{\dagger}$ Min-Kyu Cho, ${ }^{\dagger}$ Ashutosh Kumar, ${ }^{\dagger}$ Elke Maier, ${ }^{\ddagger}$ \\ Carsten Siebenhaar, ${ }^{\dagger}$ Stefan Becker, ${ }^{\dagger}$ Claudio O. Fernandez, ${ }^{\S}$ Hilal A. Lashuel," \\ Roland Benz, ${ }^{\ddagger}$ Adam Lange, ${ }^{\dagger}$ and Markus Zweckstetter ${ }^{*,+, \perp}$ \\ Department of NMR-Based Structural Biology, Max Planck Institute for Biophysical Chemistry, \\ 37077 Göttingen, Germany, DFG Research Center for the Molecular Physiology of the Brain \\ (CMPB), 37073 Göttingen, Germany, Instituto de Biología Molecular y Celular de Rosario, \\ Universidad Nacional de Rosario, Suipacha 531, S2002LRK Rosario, Argentina, Brain Mind \\ Institute, Ecole Polytechnique Federale de Lausanne (EPFL), Lausanne CH 1015, Switzerland, \\ and School of Engineering and Science, Jacobs-University Bremen, 28759 Bremen, Germany
}

Received September 12, 2009; E-mail: mzwecks@gwdg.de

\begin{abstract}
Soluble oligomers are potent toxins in many neurodegenerative diseases, but little is known about the structure of soluble oligomers and their structure-toxicity relationship. Here we prepared onpathway oligomers of the 140-residue protein $\alpha$-synuclein, a key player in Parkinson's disease, at concentrations an order of magnitude higher than previously possible. The oligomers form ion channels with well-defined conductance states in a variety of membranes, and their $\beta$-structure differs from that of amyloid fibrils of $\alpha$-synuclein.
\end{abstract}

\section{Introduction}

In many neurodegenerative diseases, such as Alzheimer's and Parkinson's disease, proteinaceous aggregates are observed in damaged neuronal regions. ${ }^{1}$ The relationship of neuronal inclusions to disease has been intensively studied and provided strong support for the importance of protein aggregation for neurodegeneration. ${ }^{1,2}$ Accumulating evidence, however, suggests that it is not the insoluble aggregates identified by light microscopy but rather soluble oligomers that are the most neurotoxic species. ${ }^{2}$ Despite their importance for neurodegeneration and for development of therapeutic treatments, little is known about the structure of soluble oligomers and their structure-toxicity relationship. ${ }^{3}$ This is due to the nature of soluble oligomers; they are intermediates of the aggregation process, and are therefore an extremely transient and labile species. $^{3}$ As soon as their concentration reaches a few percent, the so-called on-pathway oligomers are rapidly converted into amyloid fibrils, insoluble aggregates with an archetypical $\beta$-structure. In contrast, addition of small compounds, metals, lipids, and molecular chaperones to either monomeric or fibrillar protein can lead to formation of aggregation intermediates that are often stable and characterized as unordered. ${ }^{4-8}$ Soluble oligomers of the amyloid- $\beta$ peptide that were stabilized by addition of fatty acids were shown to have a $\beta$-sheet secondary

\footnotetext{
${ }^{\dagger}$ Max Planck Institute for Biophysical Chemistry.

Jacobs-University Bremen.

\& Universidad Nacional de Rosario.

"EPFL.

${ }^{\perp} \mathrm{CMPB}$.
}

(1) Taylor, J. P.; Hardy, J.; Fischbeck, K. H. Science 2002, 296, 1991-5.

(2) Haass, C.; Selkoe, D. J. Nat. Rev. Mol. Cell. Biol. 2007, 8, 101-12.

(3) Lashuel, H. A.; Lansbury, P. T., Jr. Q. Rev. Biophys. 2006, 39, 167201.

(4) Barghorn, S.; Nimmrich, V.; Striebinger, A.; Krantz, C.; Keller, P.; Janson, B.; Bahr, M.; Schmidt, M.; Bitner, R. S.; Harlan, J.; Barlow, E.; Ebert, U.; Hillen, H. J. Neurochem. 2005, 95, 834-47. structure. ${ }^{9}$ In many cases, additive-stabilized oligomers do not seed aggregation of the wild-type protein and are therefore called off-pathway. Off-pathway aggregates are believed to be nontoxic. The difficulty to produce oligomeric intermediates of defined properties in sufficient quantities has hampered efforts to (1) elucidate their structural properties, (2) develop molecular tools to monitor their formation, and (3) establish the structural basis of their toxicity.

In Parkinson's disease, aggregation of the 140 amino acid protein $\alpha$-synuclein $(\alpha S)$ is believed to be a key factor. ${ }^{10,11}$ In aqueous solution, monomeric $\alpha \mathrm{S}$ is highly flexible and belongs to the class of intrinsically disordered proteins, ${ }^{12}$ with transient long-range interactions stabilizing a closed conformation. ${ }^{13,14}$ Upon binding to negatively charged membranes, the N-terminal

(5) Behrends, C.; Langer, C. A.; Boteva, R.; Bottcher, U. M.; Stemp, M. J.; Schaffar, G.; Rao, B. V.; Giese, A.; Kretzschmar, H.; Siegers, K.; Hartl, F. U. Mol. Cell 2006, 23, 887-97.

(6) Cappai, R.; Leck, S. L.; Tew, D. J.; Williamson, N. A.; Smith, D. P.; Galatis, D.; Sharples, R. A.; Curtain, C. C.; Ali, F. E.; Cherny, R. A.; Culvenor, J. G.; Bottomley, S. P.; Masters, C. L.; Barnham, K. J.; Hill, A. F. FASEB J. 2005, 19, 1377-9.

(7) Ehrnhoefer, D. E.; Bieschke, J.; Boeddrich, A.; Herbst, M.; Masino, L.; Lurz, R.; Engemann, S.; Pastore, A.; Wanker, E. E. Nat. Struct. Mol. Biol. 2008, 15, 558-66.

(8) Kostka, M.; Hogen, T.; Danzer, K. M.; Levin, J.; Habeck, M.; Wirth, A.; Wagner, R.; Glabe, C. G.; Finger, S.; Heinzelmann, U.; Garidel, P.; Duan, W.; Ross, C. A.; Kretzschmar, H.; Giese, A. J. Biol. Chem. 2008, 283, 10992-1003.

(9) Yu, L.; Edalji, R.; Harlan, J. E.; Holzman, T. F.; Lopez, A. P.; Labkovsky, B.; Hillen, H.; Barghorn, S.; Ebert, U.; Richardson, P. L.; Miesbauer, L.; Solomon, L.; Bartley, D.; Walter, K.; Johnson, R. W.; Hajduk, P. J.; Olejniczak, E. T. Biochemistry 2009, 48, 1870-7.

(10) Lashuel, H. A.; Hartley, D.; Petre, B. M.; Walz, T.; Lansbury, P. T., Jr. Nature 2002, 418, 291.

(11) Lashuel, H. A.; Petre, B. M.; Wall, J.; Simon, M.; Nowak, R. J.; Walz, T.; Lansbury, P. T., Jr. J. Mol. Biol. 2002, 322, 1089-102.

(12) Weinreb, P. H.; Zhen, W.; Poon, A. W.; Conway, K. A.; Lansbury, P. T., Jr. Biochemistry 1996, 35, 13709-15.

(13) Dedmon, M. M.; Lindorff-Larsen, K.; Christodoulou, J.; Vendruscolo, M.; Dobson, C. M. J. Am. Chem. Soc. 2005, 127, 476-7. 
domain assumes an $\alpha$-helical structure. ${ }^{15,16}$ During aggregation $\alpha \mathrm{S}$ undergoes major conformational changes populating different oligomeric states and finally converts into a cross- $\beta$ structure, which is characteristic of amyloid fibrils. ${ }^{2}$ Solid-state nuclear magnetic resonance (NMR) spectroscopy, electron paramagnetic resonance, and quenched hydrogen/deuterium exchange revealed that the fibrillar core of amyloid fibrils of $\alpha \mathrm{S}$ comprises five $\beta$-strands within residues $35-96 .{ }^{17-19}$ In addition, a model for the fold of $\alpha \mathrm{S}$ fibrils was proposed. ${ }^{19} \mathrm{CD}$ and Fourier transform infrared spectroscopy of $\alpha \mathrm{S}$ oligomers stabilized by the aggregation inhibitor baicalin indicated the presence of $\beta$-sheet structure. ${ }^{20,21}$ In addition, fluorescence resonance energy transfer measurements pointed to conformational differences between early-stage and late-stage oligomers of $\alpha S{ }^{22}$ Detailed structural information for soluble oligomers of $\alpha \mathrm{S}$, in particular for those that are on-pathway to amyloid fibrils and are believed to be the most toxic species in Parkinson's disease, ${ }^{10}$ is missing.

Here we prepared on-pathway oligomers of $\alpha S$ at micromolar concentrations and characterized their structural and functional properties by a combination of thioflavin T (ThT) fluorescence, electron microscopy, atomic force microscopy, electrophysiological measurements, and solid-state NMR spectroscopy. The preparation method was based on our recent finding that amyloid fibrils of $\alpha \mathrm{S}$ are sensitive to low temperatures and dissociate into smaller aggregates and monomers in supercooled solution. ${ }^{23}$ We demonstrate that this allows preparation of on-pathway oligomers at concentrations an order of magnitude higher than previously possible, that the $\alpha \mathrm{S}$ oligomers form ion channels with well-defined conductance states in a variety of membranes, and that the $\beta$-structure of the oligomers is nonfibrillar.

\section{Experimental Section}

Sample Preparation for Fibrillization and Dissociation of $\alpha \mathbf{S}$ Fibrils. Recombinant $\alpha \mathrm{S}$ was expressed and purified as described. ${ }^{14}{ }^{15} \mathrm{~N}$ - and ${ }^{13} \mathrm{C} /{ }^{15} \mathrm{~N}$-labeled $\alpha \mathrm{S}$ amyloid fibrils were prepared by incubating $100 \mu \mathrm{M}$ freshly prepared ${ }^{15} \mathrm{~N}$ - and ${ }^{13} \mathrm{C} /{ }^{15} \mathrm{~N}-$ labeled $\alpha \mathrm{S}$ in a solution of $50 \mathrm{mM}$ Hepes and $100 \mathrm{mM} \mathrm{NaCl}$ at $\mathrm{pH} 7.4$ in the presence of $0.01 \%$ sodium azide in glass vials. Incubation was carried out under continuous stirring with a microsized stir bar at $37{ }^{\circ} \mathrm{C}$ until a steady state was reached according to ThT fluorescence. ${ }^{24}$ Matured fibrils were collected by centrifugation at $60000 \mathrm{rpm}(\sim 215000 \mathrm{~g})$ with a TLA 100.3 ultracentrifuge (Beckman Coulter). The collected fibrils were dissolved into distilled water and centrifuged twice to remove

(14) Bertoncini, C. W.; Jung, Y. S.; Fernandez, C. O.; Hoyer, W.; Griesinger, C.; Jovin, T. M.; Zweckstetter, M. Proc. Natl. Acad. Sci. U.S.A. 2005, 102, 1430-5.

(15) Eliezer, D.; Kutluay, E.; Bussell, R., Jr.; Browne, G. J. Mol. Biol. 2001, 307, 1061-73.

(16) Ulmer, T. S.; Bax, A.; Cole, N. B.; Nussbaum, R. L. J. Biol. Chem. 2005, 280, 9595-603.

(17) Chen, M.; Margittai, M.; Chen, J.; Langen, R. J. Biol. Chem. 2007, 282, 24970-9.

(18) Heise, H.; Hoyer, W.; Becker, S.; Andronesi, O. C.; Riedel, D.; Baldus, M. Proc. Natl. Acad. Sci. U.S.A. 2005, 102, 15871-6.

(19) Vilar, M.; Chou, H. T.; Luhrs, T.; Maji, S. K.; Riek-Loher, D.; Verel, R.; Manning, G.; Stahlberg, H.; Riek, R. Proc. Natl. Acad. Sci. U.S.A. 2008, 105, 8637-42.

(20) Hong, D. P.; Fink, A. L.; Uversky, V. N. J. Mol. Biol. 2008, 383, 214-23.

(21) Zhu, M.; Rajamani, S.; Kaylor, J.; Han, S.; Zhou, F.; Fink, A. L. J. Biol. Chem. 2004, 279, 26846-57.

(22) Kaylor, J.; Bodner, N.; Edridge, S.; Yamin, G.; Hong, D. P.; Fink, A. L. J. Mol. Biol. 2005, 353, 357-72.

(23) Kim, H. Y.; Cho, M. K.; Riedel, D.; Fernandez, C. O.; Zweckstetter, M. Angew. Chem., Int. Ed. 2008, 47, 5046-8.

(24) Hoyer, W.; Cherny, D.; Subramaniam, V.; Jovin, T. M. J. Mol. Biol. 2004, 340, 127-39. residual monomeric protein. For experiments in supercooled water at $-15{ }^{\circ} \mathrm{C}$, monomeric $\alpha \mathrm{S}(0.1 \mathrm{mM}$ protein concentration) and fibrillar $\alpha \mathrm{S}$ were suspended in $50 \mathrm{mM}$ sodium phosphate buffer $(\mathrm{pH} 7.4)$ and $300 \mathrm{mM} \mathrm{NaCl}$ and injected into glass capillaries of $0.8 \mathrm{~mm}$ inner diameter. ${ }^{23}$

Supercooling of $\alpha \mathbf{S}$ Fibrils. The cooling device (Figure S1, Supporting Information) uses an RKS cryostat with an RK20 thermostat (Lauda, Königshofen, Germany). The cooling liquid, poly(dimethylsiloxane), is directed into silicon tubes by an adjustable external pump. The tubes are connected with a jacketed cooling glass vessel with joints (Figure S1b, c), which is itself filled up with the cooling liquid. The circuit is closed by another tube leading back to the cryostat. Within the cooling vessel were placed a thermometer (to measure the temperature of the cooling liquid directly inside the vessel) and the sample, fixed by a silicon plug with two holes. Up to 100 capillaries can be placed inside the vessel. The tubes are insulated by foamed plastic. A Styrofoam box, whose holes are covered by aluminum foil, insulates the cooling vessel.

Preparation of Oligomers for Solid-State NMR Measurements. To collect a sufficient amount of $\alpha \mathrm{S}$ oligomers for solid-state NMR spectroscopy, $10 \mathrm{mg}$ of ${ }^{13} \mathrm{C} /{ }^{15} \mathrm{~N}$-labeled monomeric $\alpha \mathrm{S}$ was aggregated into amyloid fibrils and incubated at $-13{ }^{\circ} \mathrm{C}$ in the cryostat platform. After cold-induced dissociation the solution contains a mixture of monomers, oligomers, and residual fibrils/protofibrils. We removed residual fibrils/protofibrils from the solution by centrifugation in a table-top centrifuge at 5000 rpm for $15 \mathrm{~min}$. The upper $50 \mathrm{vol} \%$ of the supernatant was collected, and the lower $50 \mathrm{vol} \%$ was discarded. The collected upper portion was further centrifuged for $3 \mathrm{~h}$ at $65000 \mathrm{rpm}$ $(\sim 230000 \mathrm{~g})$, and the lower $50 \%$ portion was collected. This step was repeated several times to reduce the sample volume to 100 $\mu \mathrm{L}$. After the last centrifugation, the upper and lower $50 \%$ portions of the solution were separated and immediately freeze-trapped in liquid nitrogen. For both samples, solid-state NMR spectra were measured. $1 \mathrm{D}{ }^{13} \mathrm{C}$ cross-polarization spectra for the two samples were highly similar. Only the spectrum that was obtained for the upper $50 \%$ of the solution obtained from the last step of centrifugation is shown in Figure 7a.

Preparation of Oligomers for the Seeding Experiment. Oligomer seeds were prepared as described above except for a different starting amount of $\alpha \mathrm{S}(100 \mu \mathrm{M}$ in $300 \mu \mathrm{L})$. Neglecting any loss of oligomers during the purification procedure and considering the initial sample condition, the concentration of oligomers can have a maximum value of $\sim 500 \mu \mathrm{M}$ (in terms of monomeric $\alpha \mathrm{S}$ ) in $30 \mu \mathrm{L}$, taking into account a conversion efficiency of about $50 \%$. The purified oligomer solution $(13 \mu \mathrm{L})$ was added to $500 \mu \mathrm{L}$ of a freshly prepared solution of monomeric $\alpha \mathrm{S}$ (monomer concentration of $100 \mu \mathrm{M}$ ). Thus, the total amount of $\alpha \mathrm{S}$ in terms of monomer concentration was at most $\sim 110 \mu \mathrm{M}$ in $513 \mu \mathrm{L}$.

Atomic Force Microscopy (AFM). AFM images were recorded using an Asylum MFP-3D machine (Asylum Research, California). A $5-10 \mu \mathrm{L}$ volume of the sample solution was deposited on glass or a freshly prepared mica surface. Impurities on the glass surface were removed by plasma treatment before sample deposition. After drying in air for $1-2 \mathrm{~h}$, unbound sample and buffer were washed away with $100 \mu \mathrm{L}$ of distilled water.

Transmission Electron Microscopy. $\alpha$ S oligomers were prepared on a glow-discharged carbon foil and stained with $1 \%$ uranyl acetate. The samples were evaluated with a CM 120 transmission electron microscope (FEI, The Netherlands). Images were taken with a 2048 I 2048 TemCam 224 A camera (TVIPS, Germany) in spot mode at 195000 -fold magnification at a $-1.15 \mu \mathrm{m}$ defocus.

Polyacrylamide Gel Electrophoresis (PAGE). Conventional sodium dodecyl sulfate (SDS)-PAGE was performed with a sample buffer containing 10\% SDS. For native gel electrophoresis, 4-15\% precast polyacrylamide gels (Bio-Rad Laboratories, California) were used. 
Dynamic Light Scattering (DLS). DLS was performed with a Wyatt DynaPro Titan (Wyatt Technology, California) equipped with a temperature controller. A $12 \mu \mathrm{L}$ volume of purified oligomers at a concentration of $\sim 100 \mu \mathrm{M}$ (in terms of $\alpha \mathrm{S}$ monomers) was used. Samples were filtered with a $0.2 \mu \mathrm{m}$ syringe filter before the measurement. Hydrodynamic radius values were calculated from the acquired translational diffusion coefficient using software that comes with the DynaPro instrument.

Thioflavin T Fluorescence. Aliquots of $10 \mu \mathrm{L}$ of each sample were taken from the assay and diluted into $2 \mathrm{~mL}$ of $50 \mu \mathrm{M}$ ThT in $50 \mathrm{mM}$ glycine buffer, $\mathrm{pH}$ 8.0. ThT fluorescence spectra were measured using a Varian Cary Eclipse fluorescence spectrophotometer (Varian Inc., California) at an excitation wavelength of 446 $\mathrm{nm}$ and an emission wavelength range of 460-600 nm. Fluorescence at $480 \mathrm{~nm}$ was used for determination of the relative content of $\alpha \mathrm{S}$ fibrils in the sample. ${ }^{25}$

Solution-State NMR Spectroscopy. Solution-state NMR spectra were recorded on Bruker Avance 600 and $700 \mathrm{MHz}$ NMR spectrometers equipped with a $z$-axis gradient TXI probe (Bruker Biospin, Germany). Aggregation and dissociation of amyloid fibrils was followed by recording a series of $1 \mathrm{D}^{1} \mathrm{H}$ and $2 \mathrm{D}{ }^{1} \mathrm{H}-{ }^{15} \mathrm{~N}$ HSQC spectra. For aggregation, standard $5 \mathrm{~mm}$ NMR tubes were used. For supercooling, 10 capillaries (i.d. $0.8 \mathrm{~mm}$ ) were placed into a 5 mm NMR tube.

Quantification of $\alpha \mathbf{S}$ Oligomers. Since no direct method exists for quantification of oligomers, an indirect method was introduced. Supposing that $\alpha \mathrm{S}$ has three aggregation states-monomer, oligomer, and fibril-the amount of $\alpha \mathrm{S}$ oligomers can be estimated from the total amount of $\alpha \mathrm{S}$ by determining the relative concentrations of monomeric and fibrillar protein. The concentration of monomers present in the sample at different stages of aggregation and coldinduced dissociation was determined on the basis of the signal intensity in $2 \mathrm{D}{ }^{1} \mathrm{H}-{ }^{15} \mathrm{~N}$ HSQC spectra. The relative concentration of fibrils, on the other hand, was estimated from the ThT fluorescence intensity. The amount of protein not accounted for by either monomers or fibrils was assigned as oligomeric protein.

Solid-State NMR Spectroscopy. Solid-state NMR experiments were conducted on a 14.1 T NMR spectrometer (corresponding to a ${ }^{1} \mathrm{H}$ resonance frequency of $600 \mathrm{MHz}$ ) equipped with a $4 \mathrm{~mm}$ triple-resonance $\left({ }^{1} \mathrm{H},{ }^{13} \mathrm{C},{ }^{15} \mathrm{~N}\right)$ magic-angle spinning probe (Bruker Biospin, Germany). All experiments were carried out at a probe temperature of $-25^{\circ} \mathrm{C}$ and a magic-angle spinning rate of $8.5 \mathrm{kHz}$. Cross-polarization spectra for $\alpha \mathrm{S}$ monomers, oligomers, and fibrils were recorded using high-power proton-decoupling (SPINAL64 ${ }^{26}$ with rf amplitudes of 80-90 kHz).

Immunoblotting (Dot Blotting). $\alpha \mathrm{S}$ oligomers and amyloid fibrils were spotted onto a nitrocellulose membrane (Protran BA85, $0.45 \mu \mathrm{m}$ pore size). Blotting was performed using the conformationspecific A11 antibody (1:1000, Invitrogen's Biosource) as described previously. ${ }^{27}$ The amount of protein used for each spot was around $10 \mu \mathrm{g}$. In parallel, the samples were blotted using the anti- $\alpha \mathrm{S}$ antibody (1:2000, BD Biosciences).

Ion Channel Measurements in Planar Bilayers. Monomers, soluble oligomers, and fibrils were prepared as explained above. The membranes were formed from diphytanoylphosphatidylcholine (DiphPC) dissolved in $n$-decane. The aqueous electrolyte solutions were unbuffered and had a $\mathrm{pH}$ of $\sim 6$ unless otherwise indicated (temperature $20^{\circ} \mathrm{C}$, voltage $+100 \mathrm{mV}$ ). The average conductance, $G$, of the states (Table 1) was calculated from at least 100 single events observed with $\alpha \mathrm{S}$ oligomers in lipid bilayer experiments. Frequencies of pore formation of monomeric, fibrillar, and oligomeric $\alpha \mathrm{S}$ were determined in the following way: Three different measurement rounds were performed distributed over the time period of one year. Each round of measurement was performed

(25) LeVine, H. Methods Enzymol. 1999, 309, 274-84.

(26) Fung, B. M.; Khitrin, A. K.; Ermolaev, K. J. Magn. Reson. 2000, 142, 97-101.

(27) Kayed, R.; Head, E.; Thompson, J. L.; McIntire, T. M.; Milton, S. C.; Cotman, C. W.; Glabe, C. G. Science 2003, 300, 486-9. using freshly prepared $\alpha \mathrm{S}$ monomers, fibrils, and oligomers (starting from freshly prepared recombinant $\alpha S$ ) and consisted of 6-14 membrane preparations. Pore formation was counted as successful when (i) pores were formed at $+100 \mathrm{mV}$ or less and (ii) more than five times per preparation. Subsequently, the number of successful pore formation events was averaged over all membrane preparations.

(-)-Epigallocatechin gallate (EGCG) was obtained from SigmaAldrich, and a $10 \mathrm{mM}$ stock solution was freshly prepared in the same buffer for the experiments. For the EGCG-mixed sample, a specified volume of this EGCG solution was added to the $\alpha \mathrm{S}$ sample and the mixture was incubated at room temperature for a given time. The same measurement procedure was applied to these samples.

\section{Results and Discussion}

Preparation of Soluble Oligomers of $\alpha \mathrm{S}$ at Concentrations an Order of Magnitude Higher Than Previously Possible. Previously, cold-induced dissociation of amyloid fibrils was achieved by injection of a $300 \mu \mathrm{L}$ solution containing $\alpha \mathrm{S}$ fibrils into 10 glass capillaries, placement of the capillaries into a 5 $\mathrm{mm}$ nuclear magnetic resonance (NMR) tube, and incubation of the NMR tube at $-15{ }^{\circ} \mathrm{C}$ for one day in the vibration-free environment of an NMR spectrometer. In the present study, we replaced the NMR spectrometer by a cryostat connected to a jacketed cooling vessel (Figure S1, Supporting Information). The vessel was cooled to $-13{ }^{\circ} \mathrm{C}$ and accommodated 100 capillaries.

We followed the process of cold-induced dissociation of amyloid fibrils by transmission electron microscopy (TEM). Before cold dissociation, typical amyloid fibrils were visible (Figure 1a). After cold-induced dissociation, only a small number of fibrils and protofilaments were left and a large amount of oligomers was present (Figure 1b). Centrifugation for $15 \mathrm{~min}$ at $5000 \mathrm{rpm}$ in a table-top centrifuge and careful pipetting of the upper $50 \mathrm{vol} \%$ of the supernatant resulted in a sample in which only spherical oligomers were visible (Figure 1c). According to TEM and AFM, the spherical species were grouped into two classes with diameters of 15-20 and 20-30 $\mathrm{nm}$, respectively (Figure 1c,d). The dimensions are similar to those previously observed by TEM and AFM for on-pathway oligomers. ${ }^{3}$ Dynamic light scattering of the oligomers in solution also showed mostly two species with hydrodynamic radii of $\sim 35$ and $\sim 125 \mathrm{~nm}$ (Figure 1e). Note that the hydrodynamic radius values are only rough estimates assuming a globular shape and that EM and AFM analysis is potentially influenced by selective binding to the support. Native PAGE confirmed the presence of a large amount of soluble oligomers (Figure 1f), while SDS-PAGE showed that the oligomers are not resistant to SDS (data not shown).

A quantitative analysis of the concentration of soluble oligomers obtained by cold-induced dissociation of amyloid fibrils was performed by a combination of NMR spectroscopy and fluorescence measurements (Figure 2). Initially, a ${ }^{1} \mathrm{H}-{ }^{15} \mathrm{~N}$ two-dimensional heteronuclear correlation (HSQC) spectrum was recorded on a sample of monomeric ${ }^{15} \mathrm{~N}$-labeled $\alpha \mathrm{S}$. Next, the sample was aggregated for one week, and the concentration of remaining monomeric protein was estimated by NMR. Compared to the spectrum before aggregation, the intensity was reduced to about $15 \%$ (Figure $2 \mathrm{a}$ ). We then separated the monomeric protein from the fibrils by centrifugation and subjected the $\alpha \mathrm{S}$ fibrils to cold-induced dissociation as described previously. ${ }^{23}$ After two days of incubation in supercooled water, we determined the concentration of monomeric protein by an identical two-dimensional ${ }^{1} \mathrm{H}-{ }^{15} \mathrm{~N}$ HSQC spectrum; approxi- 

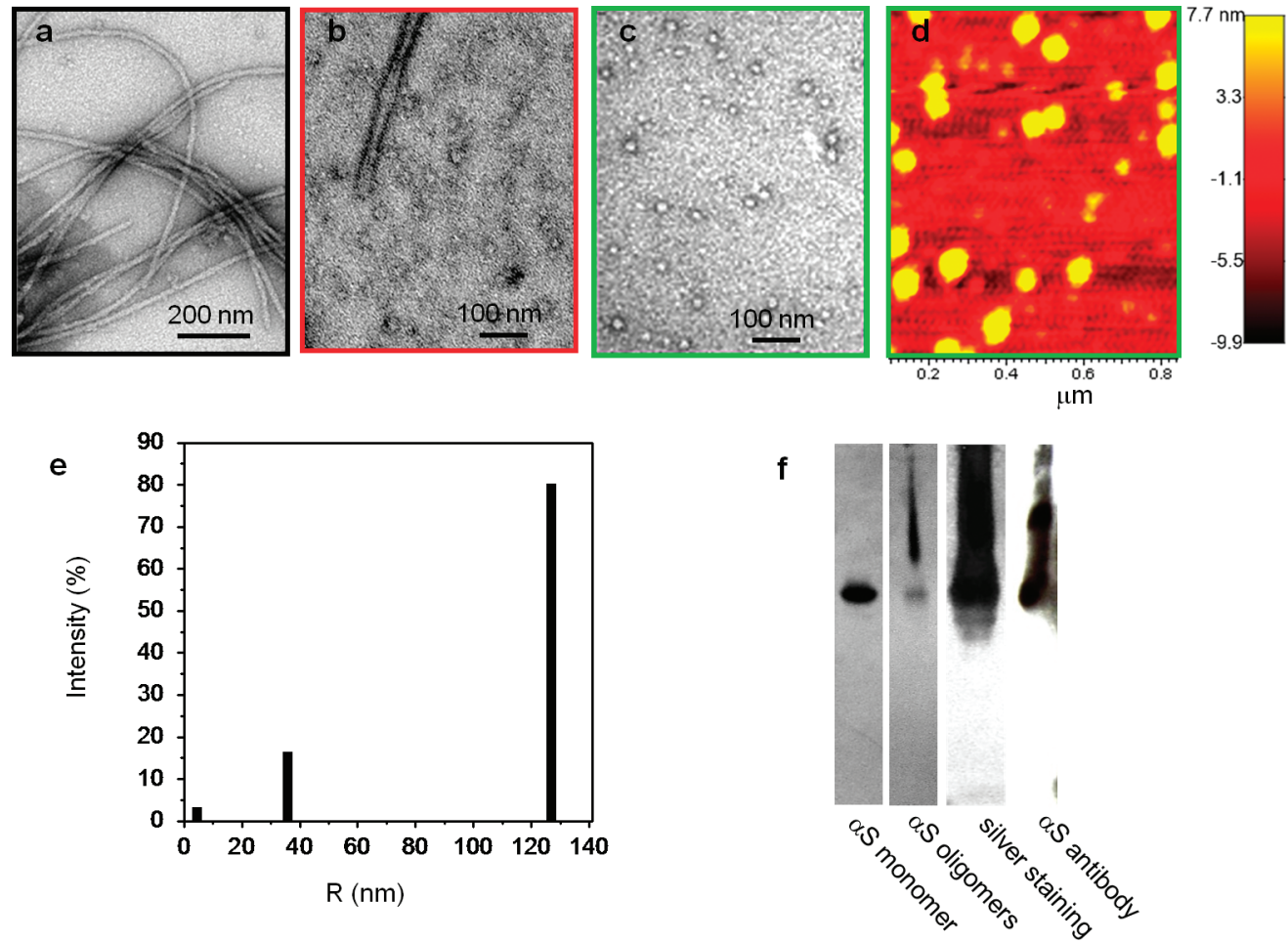

Figure 1. Morphology of $\alpha \mathrm{S}$ fibrils and soluble oligomers. (a) TEM image of freshly prepared $\alpha \mathrm{S}$ fibrils, (b) the fibrils after one day of incubation in supercooled water at $-13{ }^{\circ} \mathrm{C}$, and (c) the supernatant after centrifugation for $15 \mathrm{~min}$ at $5000 \mathrm{rpm}(\sim 2300 \mathrm{~g})$ in $50 \mathrm{mM}$ sodium phosphate buffer, $\mathrm{pH} 7.4,300$ $\mathrm{mM} \mathrm{NaCl}$. (d) AFM image of $\alpha \mathrm{S}$ oligomers. The conditions are identical to those of (c). (e) Hydrodynamic radii of the purified $\alpha \mathrm{S}$ oligomers as estimated by dynamic light scattering. Two oligomeric species were detected with sizes of $\sim 35$ and $\sim 125 \mathrm{~nm}$, respectively. Monomeric protein was also detected. (f) Native gradient (4-15\%) polyacrylamide gel of $\alpha \mathrm{S}$ monomers (lane 1) and $\alpha \mathrm{S}$ oligomers (lanes 2-4). Gels were stained with Coomasie blue (lanes 1 and 2) and silver (lane 3). Lane 4 shows the staining of the $\alpha \mathrm{S}$ oligomers using the Syn-1 antibody on a Western blot.

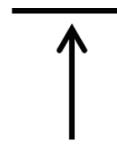

$100 \%$ monomer

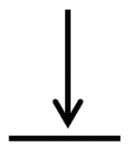

a

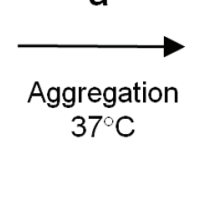

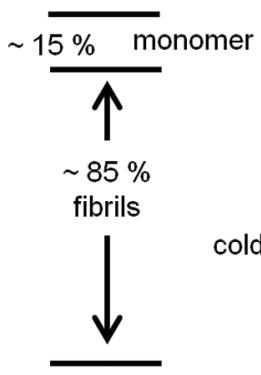

cold-induced dissociation $-15^{\circ} \mathrm{C}$

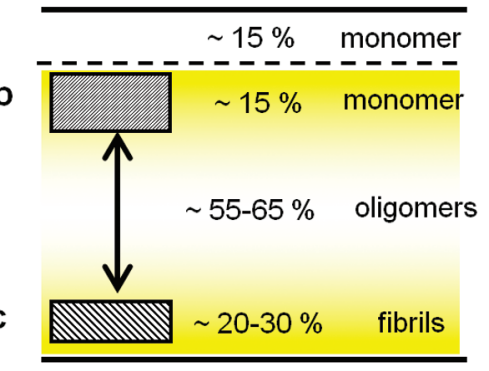

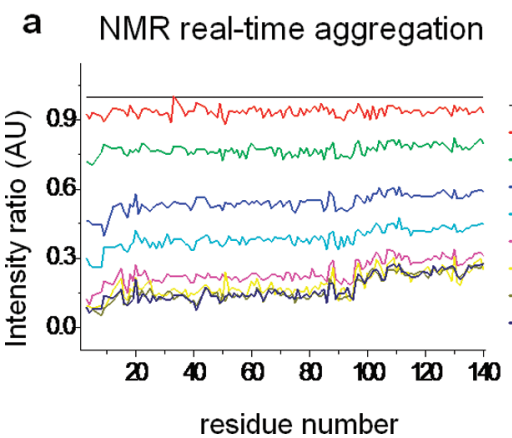

b

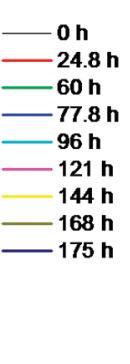

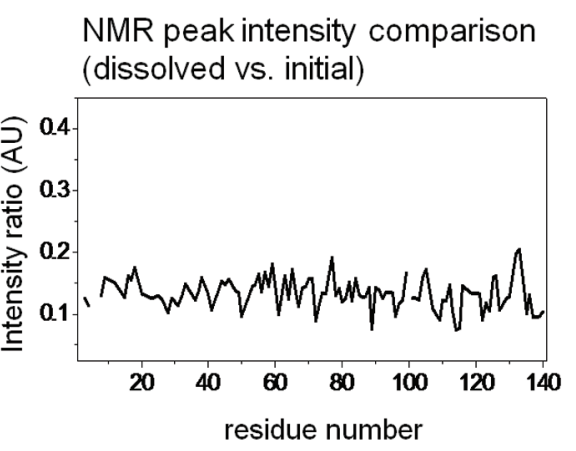

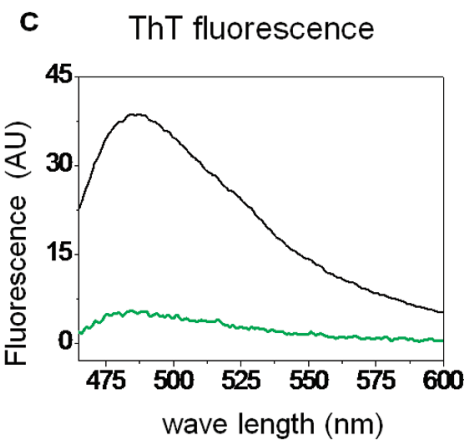

Figure 2. Quantification of $\alpha \mathrm{S}$ oligomers. (a) Decrease of NMR signal intensities of $\alpha \mathrm{S}$ residues in two-dimensional ${ }^{1} \mathrm{H}-{ }^{15} \mathrm{~N} H S Q C$ spectra during aggregation. To determine the concentration of monomeric protein before aggregation, a two-dimensional ${ }^{1} \mathrm{H}-{ }^{15} \mathrm{~N}$ HSQC spectrum was recorded on a sample of ${ }^{15} \mathrm{~N}$ labeled $\alpha \mathrm{S}$ in $20 \mathrm{mM}$ Tris, $100 \mathrm{mM} \mathrm{NaCl}, \mathrm{pH}$ 7.4. Next the sample was incubated for fibrillization. During aggregation the decrease in the concentration of monomeric $\alpha \mathrm{S}$ was monitored by two-dimensional ${ }^{1} \mathrm{H}-{ }^{15} \mathrm{~N}$ HSQC spectra. After $168 \mathrm{~h}$, the signal intensity was reduced to on average $15 \%$ when compared to the spectrum before aggregation (brown line). No additional decrease in monomer concentration was observed at $175 \mathrm{~h}$ of aggregation (dark blue line), indicating that the saturation point was reached. (b) Concentration of monomeric protein after two days of incubation in supercooled water estimated from a two-dimensional ${ }^{1} \mathrm{H}-{ }^{15} \mathrm{~N}$ HSQC spectrum. (c) Comparison of the ThT fluorescence signal before (black) and after (green) one day of incubation in supercooled water at $-13{ }^{\circ} \mathrm{C}$ suggests that $20-30 \%$ of the fibrillar material remained. The combined data demonstrate that $55-65 \%$ of the $\alpha \mathrm{S}$ fibrils were transformed into oligomeric aggregation intermediates that did not stain for the amyloid-specific dye ThT. 


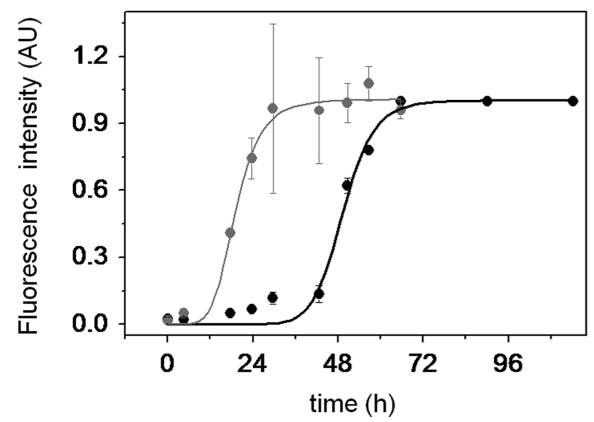

b

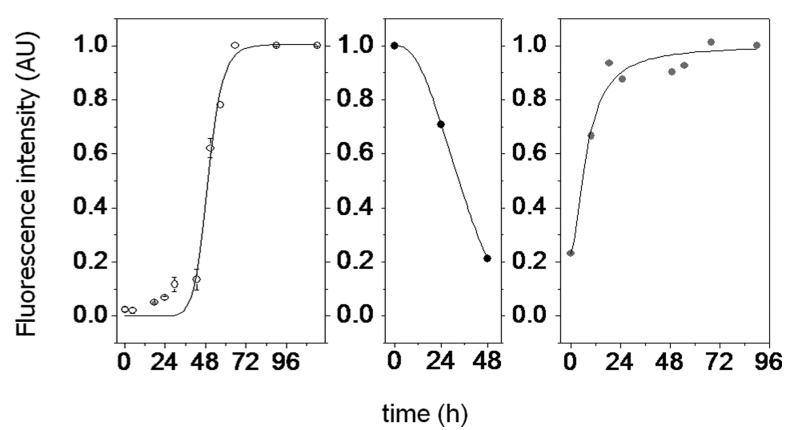

Figure 3. $\alpha \mathrm{S}$ oligomers promote aggregation. (a) ThT fluorescence assay monitoring formation of amyloid fibrils. Aggregation starting from purely monomeric $\alpha \mathrm{S}$ (black) is compared to aggregation of monomeric $\alpha \mathrm{S}$ in the presence of soluble $\alpha \mathrm{S}$ oligomers (gray). A $13 \mu \mathrm{L}$ volume of purified oligomers was added to a $100 \mu \mathrm{M} \alpha \mathrm{S}$ monomer solution prepared in the same buffer (50 mM sodium phosphate, $300 \mathrm{mM} \mathrm{NaCl}$ ). $\alpha \mathrm{S}$ oligomers dramatically accelerated fibril formation. (b) $\alpha \mathrm{S}$ aggregation and reaggregation followed by ThT fluorescence. Experiments were done in $50 \mathrm{mM}$ sodium phosphate buffer, $\mathrm{pH} 7.4,300$ $\mathrm{mM} \mathrm{NaCl}$. In the left panel, aggregation was started from monomeric $\alpha \mathrm{S}$. After $\sim 70 \mathrm{~h}$, aggregation had reached a saturation point. Subsequently, the sample was subjected to supercooling. After about two days of incubation at $-13{ }^{\circ} \mathrm{C}$, the ThT intensity dropped to about $20 \%$ of the original value (middle panel). At this stage the sample contained a mixture of $\alpha \mathrm{S}$ monomers, oligomers, and residual fibrils (Figure $1 \mathrm{~b}$ ). When the sample was warmed to $37{ }^{\circ} \mathrm{C}$ with agitation, a rapid increase in the ThT fluorescence intensity was observed (right panel).

mately $15 \%$ of the fibrils dissociated into monomeric proteins (Figure 2b). In a control measurement, $\alpha$ S fibrils were stored in capillary tubes at room temperature for two days and showed no monomeric protein in a two-dimensional HSQC spectrum. Next, we estimated the concentration of residual $\alpha \mathrm{S}$ fibrils by ThT fluorescence. Comparison of the ThT fluorescence signal before (black) and after (green) cold-induced dissociation suggested that $20-30 \%$ of the fibrillar material remained (Figure $2 c)$. The combined data demonstrate that $55-65 \%$ of the $\alpha S$ fibrils were transformed into aggregation intermediates that did not stain for the amyloid-specific dye ThT. Thus, the method of cold-induced dissociation of amyloid fibrils produces soluble oligomers at concentrations that are an order of magnitude higher than those present during aggregation.

Soluble Oligomers of $\alpha \mathrm{S}$ Dissociated from Amyloid Fibrils Are On-Pathway Aggregation Intermediates. Following quantification, we tested whether the soluble $\alpha \mathrm{S}$ oligomers are aggregation intermediates that are able to accelerate aggregation of monomeric protein. At $37^{\circ} \mathrm{C}$ with agitation, monomeric $\alpha \mathrm{S}$ showed the typical sigmoidal increase in ThT fluorescence characteristic of nucleation-dependent aggregation (Figure 3a). In contrast, addition of $13 \mu \mathrm{L}$ of the oligomer solution, which changed the total amount of $\alpha \mathrm{S}$ by less than $15 \%$, dramatically reduced the lag phase for nucleation, indicating that the $\alpha S$ oligomers are on-pathway intermediates (Figure 3a). An even more pronounced acceleration of aggregation was observed when the sample, which was obtained directly from supercooling and contained a mixture of monomers, oligomers, and fragments of fibrils, was again incubated in the aggregation conditions at $37{ }^{\circ} \mathrm{C}$ with agitation (Figure $3 \mathrm{~b}$ ). The high capacity of the purified oligomers to form fibrils and promote aggregation was further supported by the observation that purified oligomer solutions, which were stored at $4{ }^{\circ} \mathrm{C}$ without agitation in the cold room, had already formed a large amount of amyloid fibrils after two days (Figure S2, Supporting Information).

Soluble Oligomers of $\alpha \mathrm{S}$ Dissociated from Amyloid Fibrils Comprise a Neurotoxic Epitope. Little is known about the structural properties of aggregation intermediates in vivo, and antibodies are, besides dyes such as thioflavin $S$ that stain primarily amyloid fibrils, the only available tool to probe differences in the conformation of aggregation intermediates. In particular, the antibody A11 has been shown to be useful in a number of applications as it recognizes a sequence-independent

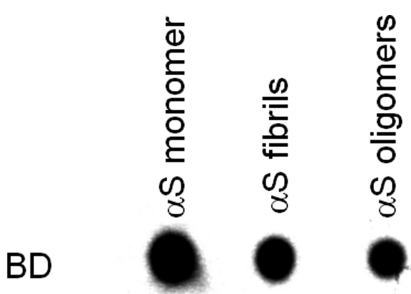

A11

Figure 4. Recognition of $\alpha \mathrm{S}$ oligomers by the $\mathrm{A} 11$ antibody. $\alpha \mathrm{S}$ monomers, fibrils, and oligomers were deposited on a nitrocellulose membrane and probed with the oligomer-specific antibody A11 and the $\alpha \mathrm{S}$-specific antibody Syn1 (BD).

structural feature common to soluble oligomers associated with neurotoxicity. ${ }^{27}$ Here we tested whether the soluble oligomers obtained by cold dissociation of amyloid fibrils of $\alpha \mathrm{S}$ and purified as described in the Experimental Section, react with the A11 antibody. Figure 4 shows that A11 stains the $\alpha \mathrm{S}$ oligomers on dot blots, but does not react with monomeric or fibrillar $\alpha S$, in agreement with previous reports. ${ }^{5,7}$ Thus, soluble oligomers of $\alpha \mathrm{S}$ dissociated from amyloid fibrils contain an epitope that is important for neurotoxicity in vivo.

Soluble $\alpha \mathrm{S}$ Oligomers Form Pores with Well-Defined Conductance States in a Variety of Membranes. Since the observation of channel activity of amyloidogenic proteins in 1993, it has been considered as a potential mechanism of toxicity in neurodegenerative diseases. ${ }^{28,29}$ Soluble oligomers became a major candidate that may react with membranes to damage key processes within the cell. ${ }^{30}$ We studied whether the soluble $\alpha \mathrm{S}$ oligomer, identified as a neurotoxic intermediate, is able to form pores in planar lipid bilayers made by the Müller-Rudin technique. ${ }^{31}$ Similarly, monomers and fibrils were also studied in a control experiment. Monomers and fibrils showed only minor or no channel-forming activity in painted lipid bilayer

(28) Arispe, N.; Rojas, E.; Pollard, H. B. Proc. Natl. Acad. Sci. U.S.A. 1993, 90, 567-71.

(29) Arispe, N.; Pollard, H. B.; Rojas, E. Ann. N.Y. Acad. Sci. 1994, 747, 256-66.

(30) Cookson, M. R.; van der Brug, M. Exp. Neurol. 2008, 209, 5-11.

(31) Benz, R.; Janko, K.; Boos, W.; Lauger, P. Biochim. Biophys. Acta 1978, 511, 305-19. 
a
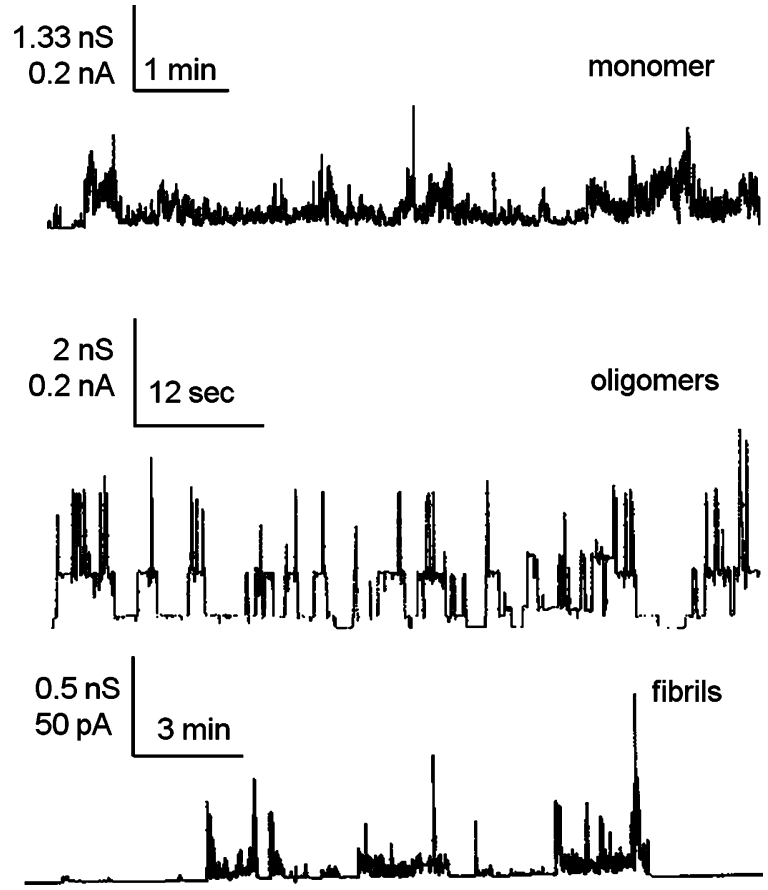

b

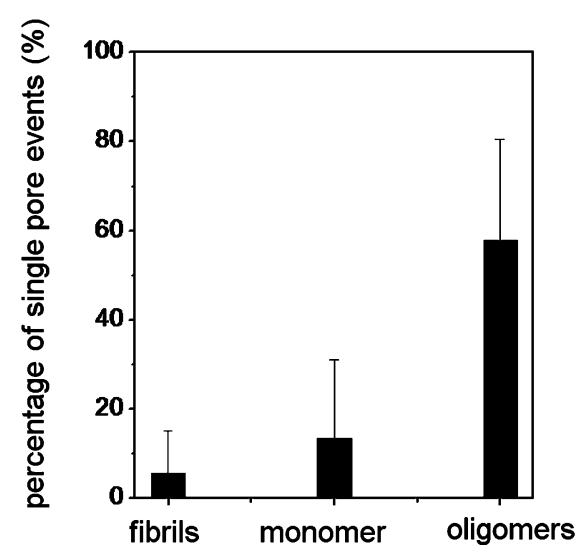

Figure 5. $\alpha$ S oligomers obtained by cold-induced dissociation of amyloid fibrils form pores in planar lipid bilayers. (a) Oligomers but not monomers or fibrils induced frequent channel formation in planar lipid bilayers formed from DiphPC/n-decane in $1 \mathrm{M} \mathrm{KCl}, 1 \%$, at $+100 \mathrm{mV}$. Oligomers were added to the cis compartment to a final concentration of $2-10 \mu \mathrm{g} / \mathrm{mL}$. (b) Frequency of pore formation for monomeric, fibrillar, and oligomeric $\alpha \mathrm{S}$ averaged over three independent sample preparations. Each measurement consisted of 6-14 membrane preparations. Successful pore formation was required to fulfill the following criteria: the pore is active (i) at $+100 \mathrm{mV}$ or less and (ii) more than five times per preparation.

membranes made from diphytanoylphosphatidylcholine dissolved to $1 \% \mathrm{w} / \mathrm{v}$ in $n$-decane (Figure $5 \mathrm{a}, \mathrm{b}$ ). In contrast, pore formation was frequently observed for the oligomers, in particular for membrane voltages of $+50 \mathrm{mV}$ and higher (Figure $5 b)$. Not only was this the case with neutral PC membranes with channel fluctuations at somewhat higher frequency, but similar conductance states were also observed for PC/PS mixtures with molar ratios of $4: 1$, i.e., with $20 \%$ negatively charged lipids probably caused by increased absorption of the positively charged $\alpha \mathrm{S}$ oligomers to the negatively charged membranes. The typical single-channel recording of Figure 5a demonstrates that soluble $\alpha \mathrm{S}$ oligomers formed many different conductance states similar to channel-forming peptides such as melittin. ${ }^{32}$ This means that the fluctuations started from a small ground conductance state of about $200 \mathrm{pS}$ and flickered at +100
Table 1. Different Conductance States Observed with $\alpha S$ Oligomers in Lipid Bilayer Experiments

\begin{tabular}{lcccc}
\hline \multicolumn{1}{c}{ salt } & ground state $(\mathrm{pS})$ & state $1(\mathrm{pS})$ & state 2 $(\mathrm{nS})$ & bursts \\
\hline $1 \mathrm{M} \mathrm{KCl}$ & 300 & 800 & 1.7 & yes \\
$1 \mathrm{M} \mathrm{LiCl}$ & 250 & 900 & 1.3 & yes \\
$1 \mathrm{M} \mathrm{KAc}(\mathrm{pH} 7)$ & 130 & 750 & 1.4 & yes \\
\hline
\end{tabular}

$\mathrm{mV}$ between different higher conductance states that were welldefined. The major conductance states at a $+100 \mathrm{mV}$ transmembrane potential were $800 \mathrm{pS}$ and about $1.7 \mathrm{nS}$ in $1 \mathrm{M} \mathrm{KCl}$ solution (Figure 5a and Table 1). An even higher conductance was observed during bursts, but these states had a very short lifetime in the millisecond range. The channels formed by the oligomers are slightly cation-selective according to measurements in different $1 \mathrm{M}$ salt solutions (Table 1). The combined data show that soluble oligomers obtained by cold-induced dissociation of amyloid fibrils of the Parkinson diseaseassociated protein $\alpha \mathrm{S}$ form ion channels with well-defined conductance states in a variety of membranes in the presence of a trans-negative potential.

The Green Tea Catechin (-)-Epigallocatechin Gallate Blocks Oligomer-Induced Pore Formation. The green tea catechin EGCG was previously shown to reduce $\alpha$ S-induced neurotoxicity in different cell models. ${ }^{33}$ The interaction of EGCG with $\alpha \mathrm{S}$ and the structural properties of EGCG-stabilized oligomers have recently been characterized by E. Wanker and colleagues. ${ }^{7}$ In this study it was shown that EGCG binds both to monomeric and to oligomeric $\alpha$ S, EGCG stimulates assembly of $\alpha \mathrm{S}$ oligomers, the EGCG-stabilized oligomers are SDS resistant, EGCG binds to the main chain of $\alpha \mathrm{S}$, and EGCGinduced oligomers are unstructured and are not recognized by the A11 antibody.

We conducted ion channel measurements with the soluble $\alpha \mathrm{S}$ oligomers and EGCG to investigate whether EGCG redirects soluble $\alpha$ S oligomers to an oligomeric species that is no longer able to form pores in planar lipid bilayers. Initially, soluble $\alpha S$ oligomers, which were obtained by cold-assisted dissociation of fibrils and were purified as described above, were incubated with increasing concentrations of EGCG for different incubation times. Subsequently, the oligomer-EGCG mixture was added to the cis compartment of the bilayer chamber system, and pore formation was monitored. When the soluble $\alpha \mathrm{S}$ oligomers were incubated with EGCG at a molar ratio of 1:10 for $2 \mathrm{~h}$, pore formation was blocked, because only insignificant channel fluctuations were observed after 2 and $12 \mathrm{~h}$ of incubation of oligomers with EGCG (Figure 6a). A reduction in the amount of added EGCG showed that a 5-fold excess of EGCG was enough to impair pore formation (Figure 6b). Thus, a molecular mechanism of the neuroprotective action of EGCG might be inhibition of pore formation resulting from soluble $\alpha \mathrm{S}$ oligomers.

Solid-State NMR Spectroscopy Indicates That the $\beta$-Structure of Soluble $\alpha \mathrm{S}$ Oligomers Is Not Fibrillar. Solidstate NMR spectroscopy is the only method that allows characterization of the structure of protein aggregates at singleresidue resolution. ${ }^{34}$ Because of the restricted sensitivity of solidstate NMR spectroscopy, however, typically as much as 0.5 $\mu \mathrm{mol}$ of isotope-labeled materials (or a $0.5 \mathrm{mM}$ concentration of the sample) is required. We tested whether the high

(32) Bechinger, B. J. Membr. Biol. 1997, 156, 197-211.

(33) Mandel, S. A.; Amit, T.; Weinreb, O.; Reznichenko, L.; Youdim, M. B. CNS Neurosci. Ther. 2008, 14, 352-65.

(34) Tycko, R. Q. Rev. Biophys. 2006, 39, 1-55. 
a
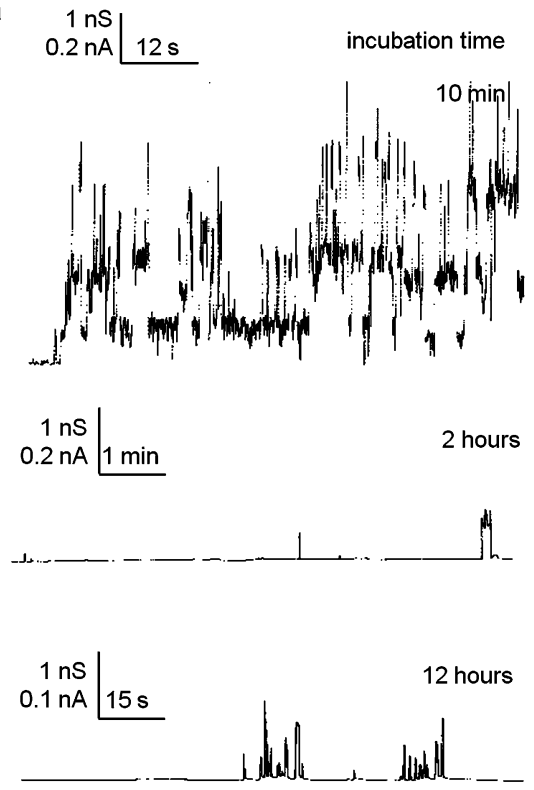

b

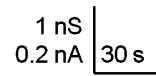

EGCG concentration

$1: 2.5$
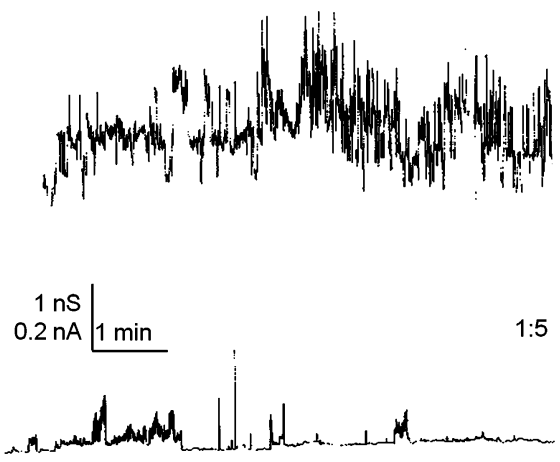

\begin{tabular}{r|r}
$1 \mathrm{nS}$ & \\
$0.2 \mathrm{nA}$ & $1 \mathrm{~min}$ \\
\hline
\end{tabular}

$1: 10$

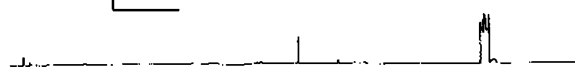

Figure 6. Inhibition of oligomer-induced pores in planar lipid bilayers by EGCG. (a) Dependence on the incubation time for a molar ratio of 1:10 oligomers: EGCG. (b) Dependence on the EGCG concentration for an incubation time of $2 \mathrm{~h}$. $\alpha \mathrm{S}$ oligomers were incubated with EGCG prior to addition to the cis compartment of the bilayer chamber. Measurements were performed for bilayers formed from DiphPC/n-decane in $1 \mathrm{M} \mathrm{KCl,} 1 \%$, at $+100 \mathrm{mV}$.
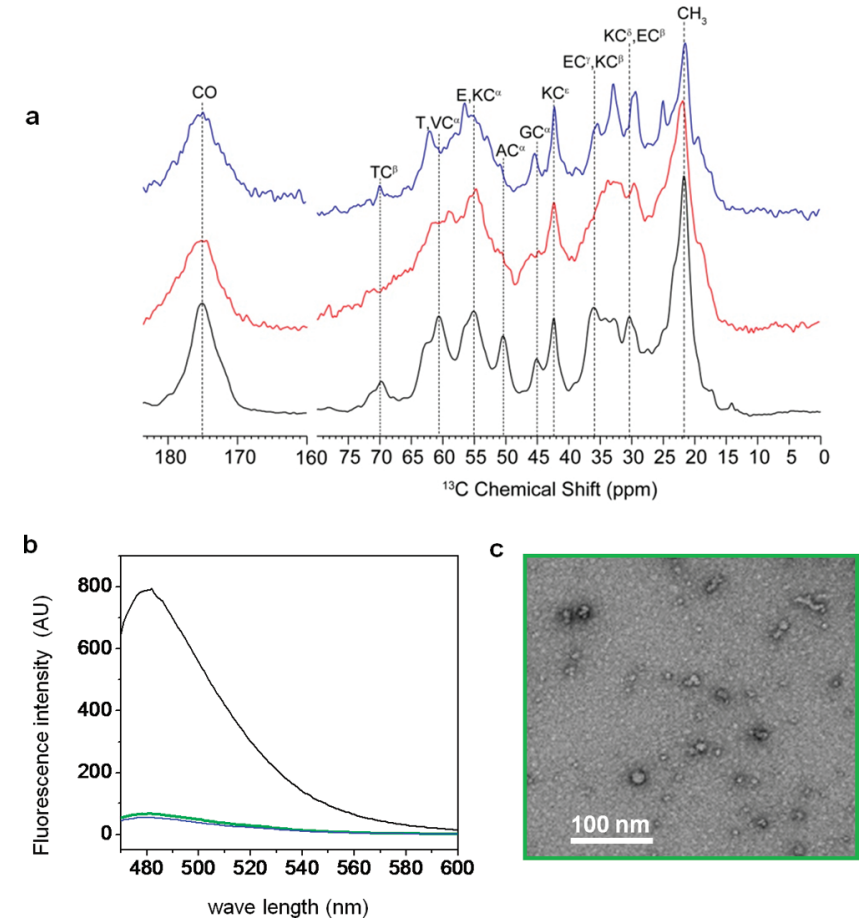

Figure 7. Solid-state NMR spectroscopy of soluble $\alpha \mathrm{S}$ oligomers. (a) ${ }^{13} \mathrm{C}$ cross-polarization solid-state NMR spectra of $\alpha \mathrm{S}$ oligomers (red), $\alpha \mathrm{S}$ monomers (blue), and $\alpha \mathrm{S}$ fibrils (black). Vertical lines are drawn on selected resonances observed in $\alpha \mathrm{S}$ fibrils. ThT fluorescence spectra (b) and electron micrographs (c) of the $\alpha \mathrm{S}$ oligomer sample directly after the solid-state NMR experiments. Emission spectra are for the blank (blue), $\alpha \mathrm{S}$ fibrils (black), and soluble $\alpha \mathrm{S}$ oligomers (green).

concentration of the soluble oligomers enables solid-state NMR measurements (Figure 7). For this aim, we prepared amyloid fibrils of ${ }^{13} \mathrm{C} /{ }^{15} \mathrm{~N}$-labeled $\alpha \mathrm{S}$, performed cold-induced dissociation of the fibrils, removed residual fragments of fibrils and protofilaments by centrifugation, and concentrated the oligomers by repeated centrifugation (see the Experimental Section). The solution remaining in the centrifuge tube contained soluble oligomers but no protofilaments or fibrils as assessed by TEM and ThT (data not shown). The consistency of the oligomer sample during the experiments was confirmed by ThT and TEM measurements, which were performed after the solid-state NMR experiments and also showed only soluble oligomers (Figure $7 \mathrm{~b}, \mathrm{c})$. The combined data suggest that the $\alpha \mathrm{S}$ oligomers were not altered during the solid-state NMR measurements.

Figure 7a compares a ${ }^{13} \mathrm{C}$ cross-polarization solid-state NMR spectrum of the $\alpha \mathrm{S}$ oligomers with spectra observed for monomeric and fibrillar $\alpha \mathrm{S}$. Monomer and oligomer samples were measured in the frozen state, whereas fibrillar $\alpha \mathrm{S}$ was measured in the nonfrozen state to exclude the contribution coming from residual embedded monomeric $\alpha \mathrm{S}$. The NMR lines observed for fibrillar $\alpha \mathrm{S}$ are quite sharp, indicative of a wellordered state. In agreement with the conversion of a natively unfolded monomeric protein into amyloid fibrils with $\beta$-structure, an upfield shift of the $\mathrm{C}^{\alpha}$ resonances of threonine, valine, glutamate, and lysine is seen for the $\alpha \mathrm{S}$ fibrils (black) when compared to the monomeric protein (blue).

The spectrum of the soluble oligomers (red) differs from both the monomeric and the fibrillar spectra. First, the NMR lines of oligomeric $\alpha S$ are broad, suggesting an intermediate structure. Second, the $C^{\prime}$ and $C^{\alpha}$ resonances of the $\alpha S$ oligomers are between those of the fibrils and monomers (Figure 7a).

Similar to oligomeric intermediates that are formed during aggregation into amyloid fibrils, the soluble oligomers obtained by cold-assisted dissociation of amyloid fibrils are not a single species. Thus, the red solid-state NMR spectrum shown in Figure 7a will include contributions from different oligomeric species. This can include contributions from monomeric protein, small fractions of oligomers with fibrillar-like $\beta$-structure, and oligomers with $\beta$-structure that differ from amyloid fibrils. At the same time, however, the NMR spectrum of the soluble oligomers (Figure 7a) is clearly not just the superposition of the NMR spectra of the monomer and the fibrils, or the spectrum of oligomers with fibrillar $\beta$-structure (as no fibrils were observed in the sample by EM, AFM, and ThT) (Figures 1 and $7 \mathrm{~b}, \mathrm{c})$. Moreover, the A11 antibody only recognizes the oligo- 
meric sample but not the monomeric and fibrillar samples (Figure 4), suggesting that the solid-state NMR spectrum reveals structural properties of soluble oligomers with a nonfibrillar structure.

How do these findings compare to structural information obtained for aggregation intermediates of the amyloid- $\beta(\mathrm{A} \beta)$ peptide and the prion protein? Ishii and colleagues determined the sequence-specific secondary structures and got some hints toward the supramolecular structure of an amyloid intermediate of the 40-residue $\mathrm{A} \beta$ peptide using solid-state NMR. ${ }^{35}$ The $\beta$-sheet structure of this late-stage aggregate was fibril-like. In contrast, liquid-state NMR showed that $\mathrm{A} \beta$ oligomers stabilized by addition of small amounts of aliphatic hydrocarbon chains of detergents had a mixed parallel and antiparallel $\beta$-sheet structure, which is different from that of fibrils. ${ }^{9}$ Solid-state NMR studies of oligomers formed by a peptide comprising residues $106-126$ of the human prion protein support formation of an extended $\beta$-strand similar to one seen in amyloid fibrils of the same peptide. ${ }^{36}$ In contrast, pulse-labeling hydrogen-deuterium exchange coupled to mass spectrometry indicated that aggregation of an SH3 domain occurs initially through relatively disordered species that subsequently evolve to form ordered aggregates that eventually lead to amyloid fibrils. ${ }^{37}$ The solidstate NMR data shown in Figure 7 are in agreement with a nonfibrillar $\beta$-structure of soluble oligomers of $\alpha \mathrm{S}$. Moreover, the observation that the soluble $\alpha$ S oligomers but not the fibrils formed pores in lipid bilayers points to a lower importance of $\beta$-structure for pathological function. In agreement with this conclusion, a recent study from us showed that $\alpha \mathrm{S}$ variants, for which the $\beta$-structure was impaired in insoluble late-state

(35) Chimon, S.; Shaibat, M. A.; Jones, C. R.; Calero, D. C.; Aizezi, B.; Ishii, Y. Nat. Struct. Mol. Biol. 2007, 14, 1157-1164.

(36) Walsh, P.; Yau, J.; Simonetti, K.; Sharpe, S. Biochemistry 2009, 48, 5779-81.

(37) Carulla, N.; Zhou, M.; Arimon, M.; Gairi, M.; Giralt, E.; Robinson, C. V.; Dobson, C. M. Proc. Natl. Acad. Sci. U.S.A. 2009, 106, 782833. aggregates, caused the most pronounced neurotoxicity in four different model systems of Parkinson's disease. ${ }^{38}$

\section{Conclusion}

Accumulating evidence suggests that soluble oligomers are potent neurotoxins in many neurodegenerative diseases. The high propensity of the soluble $\alpha \mathrm{S}$ oligomers to form pores in lipid bilayers suggests that reconstitution of $\alpha \mathrm{S}$ oligomers into proteoliposomes might be possible. The reconstituted $\alpha \mathrm{S}$ membrane pore could then be characterized at high resolution by solid-state NMR and electron paramagnetic resonance spectroscopy. The ability to prepare soluble oligomers of $\alpha \mathrm{S}$ at high concentrations is essential not only for understanding the structural basis of oligomer toxicity, but also for the development of therapeutic treatments and imaging agents for monitoring $\alpha \mathrm{S}$ oligomerization in vivo.

Acknowledgment. We thank Karin Giller for technical help, Dr. Dietmar Riedel for electron micrographs, and Dr. Nasrollah Rezai-Ghaleh and Prof. Dr. Christian Griesinger for useful discussions. This work was supported by the Max Planck Society, the Alexander von Humboldt Foundation (Follow up Program) to C.O.F., the Fonds der Chemischen Industrie to R.B., the Marie Curie IIF Fellowship within the seventh European Community Framework Programme to A.K., the Marie Curie programme Neurasyn (PITNGA-2009-238316), BMBF (Grant NGFN-Plus 01GS08190) to M.Z., and a DFG Heisenberg scholarship (Grants ZW 71/2-1 and 3-1) to M.Z.

Supporting Information Available: Diagram of the cryostat in (Figure S1) and aggregation propensity of $\alpha \mathrm{S}$ oligomers (Figure S2). This material is available free of charge via the Internet at http://pubs.acs.org.

\section{JA9077599}

(38) Karpinar, D. P.; Balija, M. B. G.; Kugler, S.; Opazo, F.; Rezaei-Ghaleh, N.; Wender, N.; Kim, H.-Y.; Taschenberger, G.; Falkenburger, B. H.; Heise, H.; Kumar, A.; Riedel, D.; Fichtner, L.; Voigt, A.; Braus, G. H.; Giller, K.; Becker, S.; Herzig, A.; Baldus, M.; Jackle, H.; Eimer, S.; Schulz, J. B.; Griesinger, C.; Zweckstetter, M. EMBO J. 2009, 28 , 3256-68. 